\title{
Effect of electromagnetic field exposure on mouse brain morphological and histopathological profiling
}

\author{
Judita Zymantiene $^{1 凶}$, Vida Juozaitiene ${ }^{2}$, Rasa Zelvyte ${ }^{1}$, Vaidas Oberauskas ${ }^{1}$, \\ Ugne Spancerniene $^{1}$, Antanas Sederevicius ${ }^{1}$, Albina Aniuliene ${ }^{3}$ \\ ${ }^{1}$ Department of Anatomy and Physiology, ${ }^{3}$ Department of Veterinary Pathobiology, \\ Faculty of Veterinary Medicine, \\ ${ }^{2}$ Department of Animal Breeding, Faculty of Animal Science, \\ Lithuanian University of Health Sciences, LT-44307, Kaunas, Lithaunia \\ judita.zymantiene@1smuni.lt
}

Received: July 2, 2019 Accepted: April 17, 2020

\begin{abstract}
Introduction: Mobile phones (MP) and other electronic and communication devices that are used daily expose users to electromagnetic fields (EMF) and contribute to an increasing incidence of neurological disorders. Brain tissue is the closest organ to the MP as it operates, thus the influence of MP radiation on brain tissue is of particular concern, although research is still inconclusive. The present study investigated the possible effect of an EMF (1,350-1,375 megahertz (MHz)) from an MP on morphological and histopathological profiles in the mouse brain. Material and methods: Healthy BALB/c mice were assigned to three equal groups (a control and two experimental groups, $n=10$ each). Experimental mice were exposed to EMFs continuously for $72 \mathrm{~h}$, those of experimental group I to a $1,350 \mathrm{MHz}$ field at a specific absorption rate (SAR) of $4.0 \mathrm{~W} / \mathrm{kg}$, and group II to a 1,375 MHz field EMF at an SAR of $4.0 \mathrm{~W} / \mathrm{kg}$. Brain segmentation and histopathological analysis were applied to detect changes in the morphometric parameters of the brain lobes and identify pathological lesions, respectively. Results: Histopathology results revealed shrinkage of pyramidal neurons, presence of mild perivascular and perineural oedema, and some vacuolation of neurons and glial cells derived from mouse great hemispheres. The lesions also included reduction of Purkinje cells, vacuolisation of neurons and glial cells, and interstitial oedema in the cerebellum. Conclusion: MP distance of $3 \mathrm{~cm}$ from the cage may induce appreciable morphological changes in mouse brain structures; therefore, more comprehensive research is essential for assessment of safe distance. These pronounced effects may interfere with the results of laboratory tests on murine experimental models in veterinary or biomedical research.
\end{abstract}

Keywords: mouse, electromagnetic field, brain, histopathology.

\section{Introduction}

Nowadays, almost all human beings are always surrounded by technology, and different intensities of electromagnetic field (EMF) exposure accompany them at home, in the workplace, at public facilities, etc. Mobile phones (MP) are an integral part of modern telecommunication systems and have become globally ubiquitous $(2,12,21)$. Currently, mobile communication shapes daily life through better connectivity and intelligent smartphone services (19). However, many animal and human studies have emphasised the deleterious biological consequences of EMF exposure from MP for health. Others have nevertheless reported no significant influence. The debate about the effect on health of EMF exposure from MP used in close proximity to the body, especially the effect on the brain, opens a new reasearch avenue for scientists.

MP technology relies on the transmission of a radio frequency $(\mathrm{RF})$ signal which generates an EMF (a type of non-ionising radiation). MP transmit and receive mainly at $800-1,800 \mathrm{MHz}$ (5). Non-ionising radiation cannot cause ionisation; however, it has been shown to produce other biological effects, for instance by heating, altering chemical reactions, or inducing electrical currents in tissues and cells. The biological effects observed on the cardiovascular, endocrine, and immune systems and on the behaviour of animals seem to be 
thermal effects of acute exposure to EMF and microwave radiation (EMF radiation with a frequency in the range of 3,000 to $300,000 \mathrm{MHz}$ ), with negligible increases of $2^{\circ} \mathrm{C}$ needed to produce these effects $(5,21)$. As to any increased risk of developing cancer after exposure to EMF or microwave fields, the evidence for such an association is extremely weak. In vitro experiments (with small animals such as mice and rats) that show abnormal cell proliferation, changes in cell membranes, and movement of ions and substances across membranes must be cautiously evaluated when potential effects of these insults on human beings are considered. It is also difficult to extrapolate to humans the observed effects on cerebral functions that relate to the behaviour of rodents, since, among other reasons, the whole brain of these small animals is uniformly exposed to the radiation, whereas the brains of people who use MP, although being exposed, receive the highest exposure only in the part closest to the handset $(13,17$, 23). Despite this fact, cognisance of the reported observations from research on animals is a prerequisite for comprehending the corresponding mechanisms in humans.

Brain tissue consists of neuronal cell bodies, their processes (dendrites and axons, myelinated or not, which form either sparse branches and arborisations or dense fibre bundles), the interconnecting extracellular brain matrix, glial cells, blood vessels, and extracellular fluid. Each of these components may have a different influence on the local mechanical properties of the tissue, which, in turn, regulate a wide variety of very relevant mechanotransduction phenomena (4). Multiple vital neural cell processes are impacted by mechanical signals, and an abnormal mechanical environment can impair brain function and neurodevelopment and progress neurological disorders (4).

Rodents (mice and especially Mus musculus and rats) are the most widely used animal models for human neurological and psychiatric disorders neurodegenerative diseases including Parkinson's disease, Huntington's disease, and Alzheimer's disease, and drug assays. The laboratory mouse and rat are an undeniable part of today's biomedical and veterinary medicine research. Mice are extensively used in physiological research (20). They are recognised as the preeminent model in numerous research fields, including neurobehavioural studies, cancer, and toxicology (24). Fagundes and Taha (10) assessed that about $85 \%$ of the articles in Medline and $70.5 \%$ of the articles in Lilacs (bibliographic databases of life sciences and biomedical information) employed mice and rats as model of various human diseases, including brain research.

The central nervous system is the main priority for EMF health research since MP usage involves close exposure or immediate contact with the head, and then the human brain is exposed to relatively high SAR of EMF when compared to other organs (5). Exposure to $900 \mathrm{MHz}$ EMF has provided evidence of causation of blood-brain barrier damage, induction of glial reactivity, and initiation of biochemical modifications in the rat brain $(17,27)$. Xu et al. (28) determined that exposure to $1,800 \mathrm{MHz}$ RF EMF promoted oxidative damage to mitochondrial DNA and changed the activity of neuron function. Dindic et al. (8) reported biochemical and histopathological changes after MP exposure on rat brain. Studies carried out by Aldad et al. (3) indicated that foetal RF radiation exposure from 800 to $1,900 \mathrm{MHz}$ affected neurodevelopment in mice. Similar results were reported by Cardis et al. (6) who noted that distribution of RF energy emitted by MP changed anatomical structures of the brain. Clinically, priority is given to epidemiological studies of the relationship between the use of MP and the incidence of brain and salivary gland tumours, acoustic neuromas, and other head and neck tumours, leukaemia, and lymphomas (12, 14, 19). Mounting evidence specifically from long-term mobile phone use (cumulative exposure) suggests that it can cause brain tumour, acoustic neuroma, and appreciable long-term deficits in learning abilities and memory functions $(9,16,23)$, and thus it, raises public concern and compels investigation.

Until now, no solid evidence has been found of the effects of EMF exposure from MP on histomorphological characteristics in the brain, and therefore, we aimed to investigate the possible adverse effect applying two different exposure levels of EMF originating from $\mathrm{MP}$ in $\mathrm{BALB} / \mathrm{c}$ strain mouse brain model.

\section{Material and Methods}

Management and experimental designs. In total, the study included 30 healthy randomly selected $\mathrm{BALB} / \mathrm{c}$ mice (15 females and 15 males from the same clutch) weighing 20-22 g, which were obtained from the vivarium and kept in standard plastic cages (Techniplast, Buguggiate, Italy). Food pellets and tap water were supplied without any restriction. The mice were maintained under a 12/12 h light/dark cycle (lights turning on at 7:00 am) at a temperature of $20-24^{\circ} \mathrm{C}$ and a relative humidity of $55 \%-65 \%$. After a 5-day adaptation period, the mice were randomly divided into a control group $(n=10)$ and two experimental groups (EMF group I and EMF group II, $\mathrm{n}=10$ each) with different exposure levels of EMF. Each group was kept in a separate cage. The mice of the control group were not exposed to EMF from MP. EMF group I was exposed to $1,350 \mathrm{MHz} \mathrm{RF}$ at a SAR of $4.0 \mathrm{~W} / \mathrm{kg}$, and EMF group II was irradiated to $1,375 \mathrm{MHz}$ RF at a SAR of $4.0 \mathrm{~W} / \mathrm{kg}$ continiously for $72 \mathrm{~h}$. The distance between $\mathrm{MP}$ and cages was $3 \mathrm{~cm}$. At the end of the experiment, the animals were sacrificed. All efforts were made to minimise the number of mice necessary for the study and their suffering. The mice were decapitated, and the brain was removed from the skull.

Morphological and histopathological studies. The skull-stripping method was applied to obtain accurate brain extraction. Mouse brain structures were 
clearly visible after opening the skull. The brains were evaluated in the standard established manner against a histological atlas (Mouse Hippocampal Atlas), available online at https://scalablebrainatlas.incf.org/mouse/ BLHS13. The evaluation of mouse brain segmentation included visual inspection, shape, and volumetric measurements. Measuring of brain lobes was performed using an FC-910 2D measuring reticule of $19 \mathrm{~mm}$ diameter and $1.5 \mathrm{~mm}$ thickness with a $60 \times 4$ cross scaleplate and 0.1 DIV, and lobes were viewed under a stereoscopic microscope. Also, morphometric parameters of the brain in the olfactory bulb (OB), superior colliculus in the septum (SC), interior colliculus in the septum (IC), cerebellum (C), medulla oblongata (MO), and olfactory bulb-cerebellum (OBC) regions were assessed. The relative brain weight (RBW) index was calculated as the ratio of brain weight to body.

After segmentation and measurement, the brain was immediately excised and fixed in $10 \%$ neutral formalin. Slices were cut in a horizontal plane with a thickness of approximately $300 \mu \mathrm{m}$. The paraffin blocks were made using Pathcentre (Thermo Shandon, Runcorn, UK) and TES 99 (Medite Medizintechnik, Burgdorf, Germany) equipment. Serial 4- $\mu \mathrm{m}$ sections were prepared from each sample with an Accu-Cut SRM microtome (Sakura Finetek, Tokyo, Japan) and underwent routine haematoxylin and eosin (H\&E) staining. Paraffin sections of $4 \mu \mathrm{m}$ in thickness were prepared for analysis of the histological and histochemical staining.

Data analysis. Statistical analysis was performed using SPSS version 15 (IBM SPSS Inc., Armonk, NY, USA). Data are presented as mean and standard deviation of mean $(\mathrm{M} \pm \mathrm{SD})$ and $95 \%$ confidence interval (CI). Differences in parameters among groups were determined using Student's paired $t$-test. Values of $\mathrm{P}<0.05$ were considered significant.

\section{Results}

The main findings of this study are presented in Tables 1 and 2 and supplemented with Figures 1-4. Accurate segmentation of the brain lobes (Table 1) clearly demonstrated that the $\mathrm{OB}$ was the greatest in size (excluding the $\mathrm{OBC}$ section, which is not a single lobe in the strict sense). The OB lobe in EMF groups I and II was respectively $0.80 \%$ and $1.40 \%$ larger than the control group. Similar results (increments of $0.97 \%$ and $3.88 \%$, respectively) were recorded in the SC lobe in EMF groups I and II. The C lobe presented enlargement of $2.45 \%$ in EMF group I and $4.09 \%$ in EMF group II compared with the control. Analogous outcomes $(1.83 \%$ and $4.09 \%$ greater size, respectively) were noticed in the MO lobe in EMF groups I and II in comparison to controls. The OBC section increased by $1.22 \%$ in EMF group II over the control animal size for this brain region, but it actually decreased by $1.10 \%$ in EMF group I. No significant differences between groups were observed analysing measurements of the IC lobe.

Segmentation of different mouse brain lobes in groups is shown in Fig.1. The OB size in EMF group I was $0.59 \%$ higher than that in the control group, whereas notable changes in OB size did not appear in EMF group II. The SC size among all groups was similar. The IC lobe presented decrements of $20.35 \%$ and $20.56 \%$ ( $\mathrm{P}<0.05)$ in EMF group I and EMF group II, respectively, compared with the control. Increments of $0.53 \%$ in group I and $0.42 \%$ in group II were recorded in the size of the $\mathrm{C}$ lobe. Similar were the findings (increments of $0.20 \%$ and $0.29 \%$, respectively) for the size of the MO lobe in EMF groups I and II.

The analysis of average body weight, brain weight, and RBW of the mice is comprised in Table 2. Brain weight decreased from that of the control group by $4.66 \%$ and $7.25 \%$ in EMF groups I and II, respectively, whereas RBW increased by $4.50 \%$ and $18.18 \%$.

Table 1. Morphometrical data of mouse brain

\begin{tabular}{llll}
\hline Lobe & $\begin{array}{l}\text { Control } \\
(\mathrm{n}=10)\end{array}$ & $\begin{array}{l}\text { EMF group I } \\
(\mathrm{n}=10)\end{array}$ & $\begin{array}{l}\text { EMF group II } \\
(\mathrm{n}=10)\end{array}$ \\
\hline & $\mathrm{M} \pm \mathrm{SD}(\mathrm{mm})$ & $\mathrm{M} \pm \mathrm{SD}(\mathrm{mm})$ & $\mathrm{M} \pm \mathrm{SD}(\mathrm{mm})$ \\
\hline OB & $5.00 \pm 0.13$ & $5.04 \pm 0.11$ & $5.07 \pm 0.08$ \\
$95 \%$ CI & $4.91-5.09$ & $4.96-5.12$ & $5.01-5.13$ \\
$\mathrm{P}$ & 0.0999973 & 0.0889386 & 0.0917641 \\
$\mathrm{SC}$ & $2.06 \pm 0.11$ & $2.08 \pm 0.11$ & $2.14 \pm 0.11$ \\
$95 \%$ CI & $1.98-2.14$ & $2.00-2.16$ & $2.06-2.22$ \\
$\mathrm{P}$ & 0.0999993 & 0.0889386 & 0.0917651 \\
$\mathrm{IC}$ & $1.98 \pm 0.04$ & $1.98 \pm 0.04$ & $1.99 \pm 0.03$ \\
$95 \% \mathrm{CI}$ & $1.95-2.01$ & $1.95-2.01$ & $1.97-2.01$ \\
$\mathrm{P}$ & 0.0836604 & 0.081163 & 0.0800674 \\
$\mathrm{C}$ & $2.44 \pm 0.16$ & $2.50 \pm 0.14$ & $2.54 \pm 0.08$ \\
$95 \% \mathrm{CI}$ & $2.33-2.55$ & $2.40-2.60$ & $2.48-2.60$ \\
$\mathrm{P}$ & 0.1241794 & 0.1266725 & 0.1280379 \\
$\mathrm{MO}$ & $1.09 \pm 0.10$ & $1.11 \pm 0.13$ & $1.15 \pm 0.14$ \\
$95 \% \mathrm{CI}$ & $1.02-1.16$ & $1.02-1.20$ & $1.05-1.25$ \\
$\mathrm{P}$ & 0.0320313 & 0.0317181 & 0.0324736 \\
OBC & $16.43 \pm 2.03$ & $16.25 \pm 2.12$ & $16.63 \pm 2.07$ \\
$95 \% \mathrm{CI}$ & $15.44-17.43$ & $14.78-17.72$ & $15.19-18.06$ \\
\hline
\end{tabular}

95\% CI - confidence interval; $\mathrm{P}$ - value; $\mathrm{OB}$ - olfactory bulb; SC - superior colliculus in the septum; IC - interior colliculus in the septum; $\mathrm{C}$ - cerebellum; $\mathrm{MO}$ - medulla oblongata; $\mathrm{OBC}$ - olfactory bulbcerebellum; EMF - electromagnetic field; $\mathrm{M} \pm \mathrm{SD}$ - mean and standard deviation of mean 


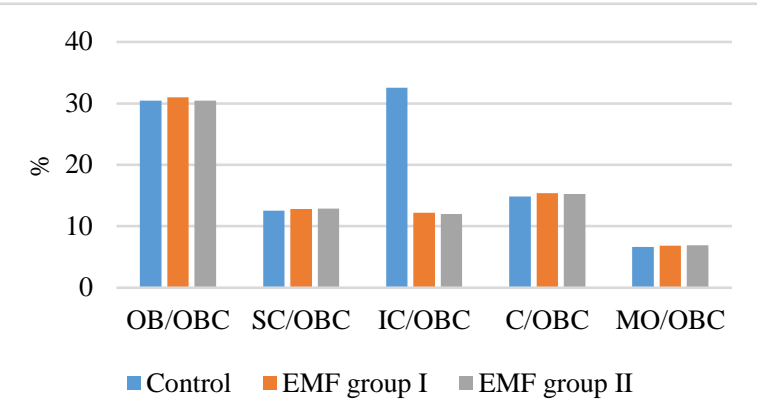

Fig. 1. Comparison of mouse brain segmentation in groups $(\%)$

Table 2. Analysis of brain weight

\begin{tabular}{lllll}
\hline Group & $\mathrm{N}$ & Body weight, $\mathrm{g}$ & Brain weight, mg & $\mathrm{RBW}$ \\
\hline & & $\mathrm{M} \pm \mathrm{SD}$ & $\mathrm{M} \pm \mathrm{SD}$ & \\
\hline Control & $\mathrm{n}=10$ & $24.00 \pm 0.00$ & $193 \pm 12.52$ & 0.22 \\
95\%CI & & $24.00-24.00$ & $184.04-201.38$ & \\
EMF I & $\mathrm{n}=10$ & $21.77 \pm 2.87$ & $202 \pm 15.63$ & 0.23 \\
95\%CI & & $19.99-23.57$ & $189.02-211.38$ & \\
EMF II & $\mathrm{n}=10$ & $18.59 \pm 3.35$ & $207 \pm 16.36$ & 0.26 \\
$95 \% \mathrm{CI}$ & & $16.52-20.66$ & $195.49-218.91$ & \\
\hline
\end{tabular}

95\% CI - confidence interval; $\mathrm{N}$ - number of animals; $\mathrm{RBW}$ - relative brain weight index; EMF - electromagnetic field; $\mathrm{M} \pm \mathrm{SD}$ - mean and standard deviation of mean

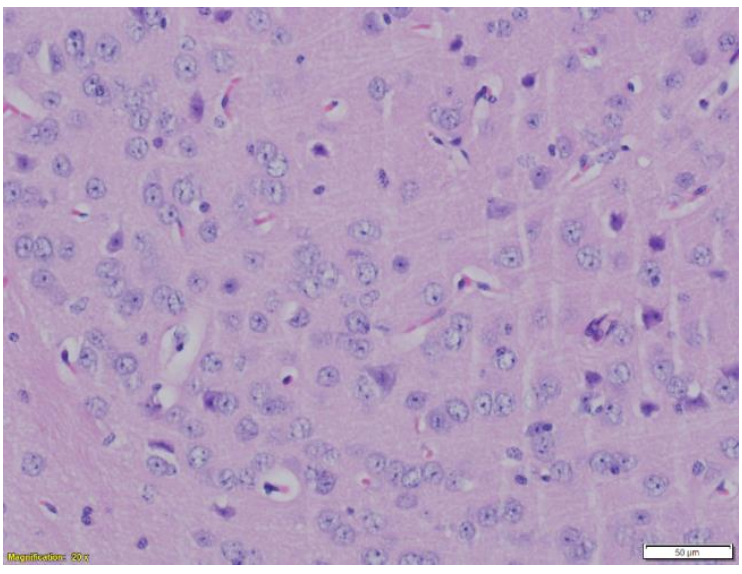

Fig. 2. Microscopic view of the great hemispheres of mice of EMF group I showing dystrophic changes (shrinkage) in some pyramidal neurons-and some vacuolation of neurons and glial cells $(\mathrm{H} \& \mathrm{E}, 200 \times)$

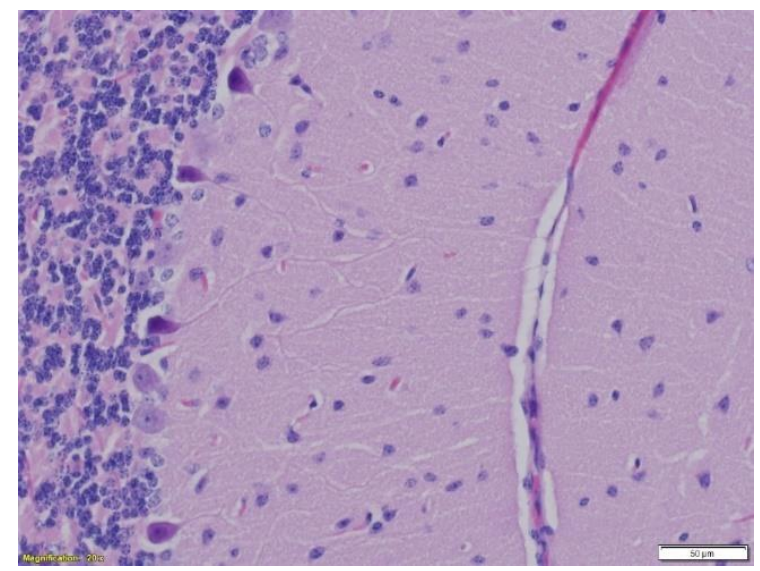

Fig. 3. Microscopic view of the cerebellum of mice of EMF group II showing dystrophic changes (shrinkage identifiable as darker, smaller examples) in Purkinje cells (H\&E, 200×)

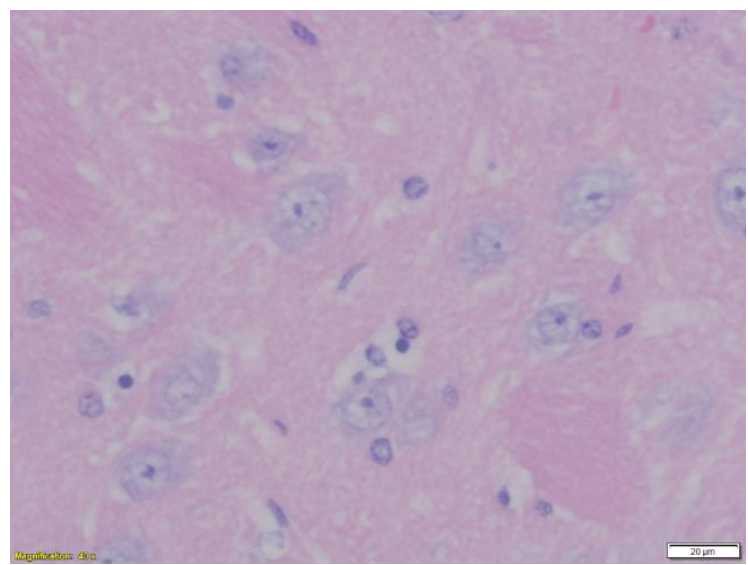

Fig. 4. Microscopic view of the vacuolated neurons and glial cells (EMF group II) and appearance of interstitial oedema (oedema in neuropil) (H\&E, 400×)

Histopathological lesions are shown in Figs 2-4. Shrinkage of pyramidal neurons, the presence of mild perivascular and perineuraloedema, and some vacuolation of neurons and glial cells derived from mouse great hemispheres were observed in EMF group I. Furthermore, reduction of Purkinje cells, vacuolisation of neurons and glial cells, and interstitial oedema in the cerebellum were also detected in EMF group II.

\section{Discussion}

Blettner and Berg (5) referred to extensive debates having persisted for decades regarding the effect of EMF exposure from MP on public health, and a large number of studies have been performed to assess potential adverse effects of that exposure. Experimental investigation suggests that RF fields are not tumour initiators and that if they were related to carcinogenicity, this would be by tumour promotion or by increasing the uptake of carcinogens in cells.

In our study, employment of two different exposure levels of EMF originating from MP had a noxious effect on mouse cortex gradients and architecture. The results obtained revealed that EMF emitted from MP changed some brain region morphological characteristics, and this affected synaptic connections and reduced microglial activation in the hippocampus.

Large observational studies performed by Singh et al. (26) suggested that regular long-term use of microwave devices (MP or microwave oven) at domestic level can have a negative impact on the body's biological systems and especially on the brain (22). A study conducted by Aberumand et al. (1) evidenced that EMF from MP has harmful effects on enzyme activity and tissue. Similar findings were noted by Kim et al. (11), who discovered that EMF exposure for 12 weeks could cause myelin damage in the cerebral cortical neuron of C57BL/6 mice. Furthermore, EMF ( $835 \mathrm{MHz}, 4.0 \mathrm{~W} / \mathrm{kg}$ SAR) exposure applied $5 \mathrm{~h}$ a day for 12 weeks rendered mice hyperactive, induced autophagy in the cortical cell body 
with significant increases in the transcriptional and protein levels of autophagy and accumulation of autophagosomes, and caused myelin damage in the cortical neurons $(3,9,11,15)$.

Our study results proved that $72 \mathrm{~h}$ EMF exposure from MP ( $3 \mathrm{~cm}$ away from body) invoked changes in the brain map of BALB/c mice, especially in the olfactory bulb, interior colliculus in the septum, and cerebellum. These changes can accelerate the rate of neuronal dysfunction associated with brain pathology.

Perepelkina et al. (20) concluded that relative brain weight was an important parameter and useable in comparative neurology. Relative brain weight changes depended on environmental conditions, physical and chemical agents, genetic factors, and rodent strain. The brain weight differences were also found to be highly significant, e.g. body weight $22.06 \pm 0.26 \mathrm{~g}$ corresponded to brain weight $438 \pm 3.66 \mathrm{mg}$ and body weight $23.48 \pm 0.73 \mathrm{~g}$ corresponded to brain weight $406.51 \pm 3.93 \mathrm{mg}$. In our study, slight differences of RBW (0.22 vs. 0.26) were observed in EMF group II after exposure to a $1,375 \mathrm{MHz}$ RF EMF.

Lessard-Beaudoin et al. (15) observed a decrease in olfactory bulb weight at 12 months of age, but our results are consistent with other rodent studies in which an increase in olfactory bulb volume with aging was noticed in Sprague-Dawley rats (18). Studies carried out by Kokturk et al. (13) indicated that exposure of rats to $900 \mathrm{MHz}$ EMF reportedly upregulates the apoptotic pathway in the cerebellum.

The outcomes of the study by Saikhedkar et al. (22) made clear a widespread neurodegeneration caused by radio waves. Increased production of reactive oxygen species due to the exhaustion of enzymatic and nonfermentative antioxidant substances and elevated lipid peroxidation indicates a significant neurodegeneration in the selective areas CA1, CA3 (pyramidal neurons in cornuammonis subfields), and dentate gyrus, as estimated using a stereotaxic atlas of the rat brain cerebral cortex. This high level of neuronal damage causes behavioural changes in animals. Furthermore, disturbance of the nervous system leads to behavioural changes and may serve as an early indicator of disturbances in regulatory functions of many systems. Behavioural changes have been noticed not just in animals but also in insects and birds (21). However, high-resolution diffusion tensor imaging, a non-invasive imaging technique used in neurodevelopment studies in transgenics or mutants, allows detection and quantification of brain structures throughout normal development in C57B/L6 mice in vivo when used at high magnetic field strength (7).

In our study, we found neurodegenerative changes in the brain. The reason for these changes may be oxidative stress or hyperthermia due to the effect of the EMF. In our case, gliocytes were frequently degenerated. It is commonly known that these cells are the main phagocytes in the brain; they are responsible for the immune response. Only in one case did we find foci of brain tissue calcination. Degenerative cells usually undergo calcination, but in our study, a very brief period of EMF wave exposure was applied, therefore such an explanation may not be correct. Notwithstanding this, according to Rafiqi et al. (21), exposure of neural tissue to EMF can cause electrophysiological changes in the nervous system such as efflux of calcium ions from brain tissue. The nonsystematic and sudden calcium efflux can manifest in many irregularities of neuronal control as calcium ions play an important role in the functions of the nervous system, part of which is the release of neurotransmitters.

This study is an analysis of complex findings associated with brain cortex changes which had never been investigated before. An understanding of the aspects analysed in this article contributes to effective protection against deleterious biological consequences of MP EMF exposure for public health.

In conclusion, different exposure levels of EMF from MP over $72 \mathrm{~h}$ affected mouse brains and had a negative influence on segmentation parameters of mouse brain structures. These findings suggested that EMF equipment operating at a $3 \mathrm{~cm}$ distance from the cage may induce appreciable morphological changes in the brain. The research outcomes enrich knowledge of neurobiological mechanisms of brain scaling, neurodegerative changes, and integration of brain mapping algorithms and recommend using the mouse as an experimental model in neuroscientific, medical, or pharmacological studies. Moreover, the findings gained have potential to yield solutions improving public health.

Conflict of Interest Statement: The authors declare that there is no conflict of interests regarding the publication of this article.

Financial Disclosure Statement: The authors declare that they did not receive any financial support and that the article was self-funded.

Animal Rigts Statement: All animal procedures were approved by the Ethics Commission of Lithuania on the Use of Laboratory Animals under the State Food and Veterinary Service (Decision no. G2-73/2017 of 3 November), and conducted in accordance with the guidelines set by the European Commission Directive 2010/63/EEC.

\section{References}

1. Aberumand M., Mansouri E., Pourmotahari F., Mirlohi M., Abdoli Z.: Biochemical and histological effects of mobile phone radiation on enzymes and tissues of mice. Res J Pharm Biol Chem Sci 2016, 7, 1962-1971.

2. Adibzadeh F., Bakker J.F., Paulides M.M., Verhaart R.F., van Rhoon G.C.: Impact of head morphology on local brain specific absorption rate from exposure to mobile phone radiation. Bioelectromagnetics 2015, 36, 66-76. 
3. Aldad T.S., Gan G., Gao X.B., Taylor H.S.: Fetal radiofrequency radiation exposure from $800-1,900 \mathrm{mhz}$-rated cellular telephones affects neurodevelopment and behavior in mice. Sci Rep 2012, 2, 1-8, doi: 10.1038/srep00312.

4. Antonovaite N., Beekmans S.V., Hol E.M., Wadman W.J., Iannuzz D.: Structure-stiffness relation of live mouse brain tissue determined by depth-controlled indentation mapping. Biol Physics 2018, arXiv: 1802.02245v1 (physics.bio-ph).

5. Blettner M., Berg G.: Are mobile phones harmful? Acta Oncol 2000, 39, 927-930.

6. Cardis E., Deltour I., Mann S., Moissonnier M., Taki M., Varsier N., Wake K., Wiart J.: Distribution of RF energy emitted by mobile phones in anatomical structures of the brain. Phys Med Biol 2008, 53, 2771-2783.

7. Chahboune H., Ment L.R., Stewart W.B., Ma X., Rothman D.L., Hyder F.: Neurodevelopment of C57B/L6 mouse brain assessed by in vivo diffusion tensor imaging. NMR Biomed 2007, 20, 375-382.

8. Dindic B., Sokolović D., Krstić D., Petković D., Jovanović J., Muratović M.: Biochemical and histopathological effects of mobile phone exposure on rat hepatocytes and brain. Acta Med Median 2010, 49, 37-42.

9. Ellenbroek B., Youn J.: Rodent models in neuroscience research: is it a rat race? Dis Model Mech 2016, 9, 1079-1087.

10. Fagundes D.J., Taha M.O.: Choices criteria and current animal specimens. Acta Cir Bras 2004, 19, 59-65.

11. Kim J.H., Da HY., Huh Y.H., Lee E.H., Kim H.G., Kim H.R.: Long-term exposure to $835 \mathrm{MHz}$ RF-EMF induces hyperactivity, autophagy, and demyelination in the cortical neurons of mice. Sci Rep 2017, doi: 10.1038/srep41129.

12. Krewski D., Byus C.V., Glickman B.W., Lotz W.G., Mandeville R., McBride M.L., Prato F.S., Weaver D.F.: Recent advances in research on radiofrequency fields and health. J Toxicol Environ Health 2001, 4, 145-159.

13. Kokturk S., Yardimoglu M., Celikozlu S.D., Dolanbay E.G., Cimbiz A.: Effect of Lycopersicon esculentum extract on apoptosis in the rat cerebellum, following prenatal and postnatal exposure to an electromagnetic field. Exp Ther Med 2013, 6, $52-56$.

14. Lahkola A., Auvinen A., Raitanen J., Schoemaker M.J., Christensen H.C., Feychting M., Johansen C., Klaeboe L., Lönn S., Swerdlow A.J., Tynes T., Salminen T.: Mobile phone use and risk of glioma in 5 North European countries. Int $\mathrm{J}$ Cancer 2007, 120, 1769-1775.

15. Lessard-Beaudoin M., Laroche M., Demers M.J., Grenier G., Graham R.K.: Characterisation of age-associated changes in peripheral organ and brain region weights in C57BL/6 mice. Exp Gerontol 2015, 63, 1-28.

16. Lönn S., Ahlbom A., Hall P., Feychting M.: Long-term mobile phone use and brain tumor risk. Am J Epidemiol 2005, 161, $526-535$.
17. Mausset-Bonnefont A.L., Hirbec H., Bonnefont X., Privat A., Vignon J., de Sèze R.: Acute exposure to GSM 900-MHz electromagnetic fields induces glial reactivity and biochemical modifications in the rat brain. Neurobiol Dis 2004, 17, 445-454.

18. Mirich J.M., Williams N.C., Berlau D.J., Brunjes P.C. Comparative study of aging in the mouse olfactory bulb. J Comp Neurol 2002, 454, 361-372.

19. Meena J.K., Verma A., Kohli C., Ingle G.K.: Mobile phone use and possible cancer risk: Current perspectives in India. Indian J Occup Environ Med 2016, 20, 5-9.

20. Perepelkina O.V., Golibrodo V.A., Lilp I.G., Poletaeva I.I.: Mice selected for large and small brain weight: The preservation of trait differences after the selection was discontinued. Adv Biosci Biotechnol 2013, 4, 1-8.

21. Rafiqi S.I., Kumar S., Chaudhary R., Farooq U.B., Kirthika P.: Mobile phone radiations and its impact on birds, animals and human beings. Trends Vet Anim Sci 2016, 3, 24-27.

22. Saikhedkar N., Bhatnagar M., Jain A., Sukhwal P., Sharma Ch., Jaiswal N.: Effects of mobile phone radiation $(900 \mathrm{MHz}$ radiofrequency) on structure and functions of rat brain. Neurol Res 2014, 36, 1072-1079.

23. Schoemaker M.J., Swerdlow A.J., Ahlbom A., Auvinen A., Blaasaas K.G., Cardis E., Christensen H.C., Feychting M., Hepworth S.J., Johansen C., Klaeboe L., Lönn S., McKinney P.A., Muir K., Raitanen J., Salminen T., Thomsen J., Tynes T.: Mobile phone use and risk of acoustic neuroma: results of the interphone case-control study in five North European countries. Br J Cancer 2005, 93, 842-848.

24. Sengupta P.: Environmental and occupational exposure of metals and their role in male reproductive functions. Drug Chem Toxicol 2012, 36, 353-368.

25. Sengupta P.: The laboratory rat: relating its age with human's. Int J Prev Med 2013, 4, 624-630.

26. Singh S., Brocker C., Koppaka V., Chen Y., Jackson B.C., Matsumoto A., Thompson D.C., Vasiliou V.: Aldehyde dehydrogenases in cellular responses to oxidative/electrophilic stress. Free Radic Biol Med 2013, 56, 89-101.

27. Tang J., Zhang Y., Yang L., Chen Q., Tan L., Zuo S., Feng H., Chen Z., Zhu G.: Exposure to $900 \mathrm{MHz}$ electromagnetic fields activates the mkp-1/ERK pathway and causes blood-brain barrier damage and cognitive impairment in rats. Brain Res 2015, 1601, 92-101.

28. Xu S., Zhou Z., Zhang L., Yu Z., Zhang W., Wang Y., Wang X., Li M., Chen Y., Chen C., He M., Zhang G., Zhong M.: Exposure to $1800 \mathrm{MHz}$ radiofrequency radiation induces oxidative damage to mitochondrial DNA in primary cultured neurons. Brain Res 2010, 1311, 189-196. 